\section{A Case Report of Organizing Pneumonia /IgG4 Related Diseases}

\section{Abstract}

Organizing pneumonia form of idiopathic interstitial pneumonia characterized by lung inflammation and scarring that obstructs the small airways and air sacs of the lungs (alveoli). Organising pneumonia is defined pathologically by the presence in the distal air spaces of buds of granulation tissue progressing from fibrin exudates to loose collagen containing fibroblasts. Since organising pneumonia is a non-specific inflammatory pulmonary process, it may result from a number of causes. Pathologists may report features of organising pneumonia in association with conditions such as infectious pneumonia, lung abscess, empyema, lung cancer, bronchiectasis, broncholithiasis, chronic pulmonary fibrosis, aspiration pneumonia (giant cells and foreign bodies usually are present), adult respiratory distress syndrome, pulmonary infarction, and middle lobe syndrome.

This is an interesting, yet challenging case trying to formulate a diagnosis of a patient presented with worsening of dyspnea over more than a year. The patient has history of unsuccessful treatment of eosinophilic asthma with biological agents while also on treatment with prednisone for query organizing pneumonia.

Keywords: Asthma; Pneumonia; IgG4; Lung diseases

\section{Naveen Dogra* \\ Department of Pulmonary Medicine, Royal Alexandra Hospital, Edmonton, Canada}

Corresponding author: Naveen Dogra

” dogranaveen77@gmail.com

Department of Pulmonary Medicine, Royal Alexandra Hospital, Edmonton, Canada

Tel: 17802464517

Citation: Dogra N (2019) A Case Report of Organizing Pneumonia /IgG4 Related Diseases. J Clin Gastroenterol Hepatol Vol.5 No.3:126

Received: September 26, 2019; Accepted: November 14, 2019; Published: November 21, 2019

\section{Introduction}

Eosinophilic asthma is a form of asthma associated with high levels of a white blood cells called eosinophils and is one of the leading causes of severe asthma. High levels of eosinophils can cause inflammation in airways, affecting the sinuses and nasal passages as well as the lower airways. The result is production of symptoms including shortness of breath, wheezing, tightness in chest, coughing, obstructed airflow, stuffy nose, nasal discharge, chronic sinus infection, and nasal polyps. Adult onset eosinophilic asthma requires systemic glucocorticoids, preferably early in the course of the disease, to maintain control of their asthma [1-4].

Organizing pneumonia is a type of diffuse interstitial lung disease that affects the distal bronchioles, respiratory bronchioles, alveolar ducts, and alveolar wall [5-8]. The primary area of injury is within the alveolar wall. Its incidence is six to seven cases $/ 100000$ hospital admissions at major teaching hospitals in Canada [9]. In general, the severity of illness at presentation is related to potential for subsequent relapse; severity of hypoxemia, extent of radiographic abnormalities, severity of the decline in lung function, presence of an underlying illness, or length of delay in beginning treatment $[10,11]$.

Conditions associated with the histopathology of organizing pneumonia [12,13];

- Cryptogenic organizing pneumonia

- Drug toxicity; amiodarone, beta blockers, bleomycin, cocaine, carbamazepine, phenytoin etc.

- Chronic heart or renal failure

- Chronic thyroiditis

- Rheumatic diseases

- Mixed cryoglobulinemia

- Hematologic malignancy

- Immunodeficiency syndromes; common variable immunodeficiency

- Infections (organizing)

- Inflammatory bowel disease 
- Inhalation injury; aspiration of gastric contents, sulfur dioxide

- Irradiation injury; breast cancer

- Reaction around other lung processes; abscess, diffuse alveolar hemorrhage, lung tumour

- Transplantation; allogenic hematopoietic cell, liver, lung

- Associated with other interstitial pneumonias; chronic eosinophilic pneumonia, hypersensitivity pneumonitis, acute respiratory distress syndrome etc.

\section{Case Report}

This case is about a 69-years old non-smoker, non-diabetic, female whose symptoms started when she was a teenager and continued to have recurrent episodes of bronchitis. She was treated initially with benadryl, but later experienced an allergic reaction, resulting in a rash. The treatment for her bronchitis attacks was switched to prednisone from which she experienced relief until she was diagnosed with asthma in her 20s. Eventually, she was started on theophylline, Ventolin, and Advair.

Starting March 2017 to April 2018, her cough became worse and was treated with multiple courses of antibiotics. In April 2018, however, she admitted herself to the hospital as she was feeling weak with low energy. She was eventually discharged with inhalers and a short course of prednisone and was scheduled to get an allergic test done. The test results were negative for any allergies.

In May 2018, the patient visited a naturopath on the recommendation of her friend as an alternative medicine to remedy her dyspnea and cough. She was informed that she had allergies to popular trees, latex and she had mould in her body. She was given natural products in the form of drops for few months by the naturopath with a scheduled follow up to asses her allergies. During her next visit, she was told that repeated tests showed no more mould in her body and advised to stop using natural products. After a few days, her symptoms of cough and weakness got worse and she went back to see her naturopath. This time he advised her to adopt a healthy lifestyle and to eat healthy food with no further treatment.

During this period, her family was also under considerable stress as the patient was not getting better and her family was pressuring her to see a specialist. She had another allergy test which also came back negative, but with the wheezy chest and Pulmonary Function Tests (PFTs) showing evidence of severe airflow obstruction with FEV1 (Forced Expiratory Volume in the first second) of $33 \%$ and with no significant change in FVC (Forced Vital Capacity) or FEV1 post bronchodilators, she was started on LAMA (long-acting muscarinic antagonists), LABA (long-acting beta-agonist), and ICS (inhaled corticosteroid) inhalers.

The patient was finally able to see a pulmonologist in July 2018. She had a chest CT (computed tomography) for progressive dyspnea and it showed mildly enlarged lymph nodes within both hila, subcarinal space, lower left and right paratracheal space. There was also evidence of a $9 \mathrm{~mm}$ ground- glass attenuating nodule within the apico-posterior segment of the left upper lobe laterally, small up to $5 \mathrm{~mm}$ mean transverse diameter noncalcified solid nodule within both lower and upper lobes. Her peripheral blood counts showed increased eosinophilia [14,15] at 1.5 (reference range 0.0-0.7) and she appeared to have high eosinophilia peripherally since May 2017. Her IgE (immunoglobulin E) level was also high at 871 (reference range $<120 \mathrm{KU} / \mathrm{L}$ ), C4 (complement 4) was low at 0.04 (reference range $0.18-0.36 \mathrm{~g} / \mathrm{L}$ ), and ANCA (antineutrophil cytoplasmic antibodies), anti-dsDNA (anti-double standard DNA), ENA (extractable nuclear antigen), ANA (antinuclear antibody), RA (rheumatoid arthritis) factor were all negative including normal IgG, IgA, and IgM. She was diagnosed to have eosinophilic asthma and was started on Benralizumab [16-18] $30 \mathrm{mg}$ subQ every 4 weeks for the first three doses, and then once every 8 weeks. While on this treatment, the patient travelled to the United States of America and she reported feeling better during this time. However, upon her return to Canada in Nov 2018, symptoms reappeared with increased shortness of breath, so it was decided that Benralizumab was not helping her, so it was discontinued.

CT scan of her chest was repeated in early December 2018. It showed the previously seen $9 \mathrm{~mm}$ ground glass nodule decreased in size to $7 \times 3 \mathrm{~mm}$, but there was also evidence of multiple small illdefined nodules bilaterally in range of $6 \mathrm{~mm}$ or less, and an $8 \mathrm{~mm}$ nodule at the medial right lower lobe. It also showed ill-defined foci of ground-glass opacity and mild consolidation within medial right lower lobe, mild mediastinal and hilar lymphadenopathy. In addition, CT finding of mild pleural nodularity and broncho vascular nodularity raise the concern for sarcoidosis. She had another CT scan of the chest in early January 2019 which showed persisting mild mediastinal and hilar lymphadenopathy with progression in numerous small pulmonary nodules bilaterally with the possibility of sarcoidosis.

The patient was referred for a bronchoscopy with CT scan finding of adenopathy with query sarcoidosis. She had her first bronchoscopy with EBUS (endobronchial ultrasound) in midJanuary 2019 and samples were sent for cytology, flow cytometry, and microbiology. The surgical pathology report showed no evidence of granulomatous inflammation or malignancy and flow cytometry showed no abnormality of $\mathrm{T}$ or $\mathrm{B}$ cells detected with $\mathrm{T}$ cell accounting for $74 \%$ with a CD4/CD8 ratio of 5.4 .

During the first bronchoscopy, the procedure was stopped before sufficient samples could be taken because of desaturation and difficulty with sedation. She was refereed for revision of bronchoscopy with EBUS for the second time during late January 2019. Unfortunately, this time again, it was a difficult procedure as her blood pressure elevated and she got agitated, was trying to pull scope the out. The procedure was terminated in between, but they were able to get mucosal biopsies with bronchial wash samples. These samples were unable to demonstrate any specific findings and even bronchoalveolar lavage cell count showed $0 \%$ eosinophil, $10 \%$ lymphocytes, $14 \%$ neutrophils, $21 \%$ epithelial cells, and $55 \%$ histiocytes.

The patient was planned to repeat bronchoscopy for transbronchial biopsy as her trachea showed white appearing 
polypoid lesions seen on the posterior surface in distal 1/3 of the trachea, endobronchial biopsy and bronchoalveolar lavage at the end of January 2019. This time also, tissue showed only chronic inflammation with no granuloma, no malignancy, and no lymphoproliferative disorder. The tracheal mucosal biopsy was consistent with inflammatory poly. The biopsy samples were further sent to Cross Cancer Institute (CCI) for review and it was recommended that there was no evidence of any lymphoproliferative disorder.

The patient had a repeated CT scan of the chest in March 2019 that still showed enlarged intrathoracic lymph node with no change from previous results in January 2019 but it did show asymmetrical, vaguely midlung zone predominant nodularity had progressed and a new small juxta pleural focus of consolidation had developed within the right lower lobe with possibilities of sarcoidosis, a lymphoproliferative disorder, or atypical infection.

The patient had three bronchoscopy procedures in January with no definite diagnosis, but still, she was well determined to undergo further investigations to have a diagnosis. She was in touch with her pulmonologist who planned for her to have lung biopsy as an outpatient procedure, but the patient became very sick with hypoxia with increase shortness of breath and was admitted to hospital in May 2019. The repeated CT scan of the chest showed multiple bilateral solid and ground glass pulmonary nodules, changed in size and distribution from the previous study. The largest measured $5 \mathrm{~mm}$ in diameter in the apical left lower lobe, but in particular, previously seen anterior left upper lobe nodule seen in January 2019 measuring $4 \mathrm{~mm}$ has resolved and the posterior left upper lobe subpleural nodule seen in March 2019 has decreased in size from $8 \mathrm{~mm}$ to $5 \mathrm{~mm}$ with stable hilar and mediastinal lymphadenopathy. ACE (Angiotensin Converting Enzyme) level was high at 86, so there was a concern of possible sarcoidosis. She was planned for an open lung biopsy during this admission. The initial results of the biopsy showed a finding of centrilobular nodules consisting of plugs of organizing pneumonia $[19,20]$ with marked lymphoplasmacytic infiltrate as well as intense eosinophilic infiltrate. There was a vascular wall infiltration with inflammatory cells, but no definite obliterative vasculopathy or necrotizing vasculitis. Immunohistochemistry showed IgG4: IgG ratio of plasma cells in the range of $40-50 \%$, and the number of IgG-positive plasma cells $[21,22]$ in the range of 60-70 per high power field which raised the possibility of IgG4-related lung disease. There was no evidence of pulmonary sarcoidosis and lymphoproliferative disorder. For the possibility of IgG4 diseases, results of the biopsy were reviewed with $\mathrm{CCl}$ and it was recommended that there is no evidence of IgG4 [23] diseases. Likely, it shows the reactive lymphoplasmacytic process as the plasmacytic cells are polytypic and most are positive for IgG, and IgG4 are rare. She was discharged home with a slow tapering of chronic prednisone [24] with the possibility of query organizing pneumonia. From recent communication with patient, she is on $20 \mathrm{mg}$ of prednisone and as far as her breathing is concerned, she feels much better now.

During the routine investigation in June 2019, her urine showed significant proteinuria with significant albumin/creatinine ratio. She had a renal biopsy in last week of July 2019 that showed features consistent with membranous nephropathy, [25] global glomerulosclerosis, severe arteriosclerosis and mild arteriolar hyalinosis. Her anti-GBM was negative at 5.7, IgG subgroup showed IgG1 3.87, IgG2 1.82, IgG3 0.12, \& IgG4 0.53. Her HIV serology, Hepatitis B surface antigen and Hepatitis C antibody were nonreactive.

Her lung biopsy samples were referred again by her pulmonologist to another institute for second opinion which she had in May 2019. It was recommended that there are features suggestive of reactive airway disease, follicular bronchiolitis and bronchocentric perivascular and sub-pleural foci of organizing pneumonia. Evidence of perivascular inflammation and involvement of vascular walls represent secondary vasculitis rather than primary vasculitis. The presence of poorly formed foci of granulomatous inflammation, centered on airways, having foamy macrophages raise the concern of aspiration [26]. Other possibilities which can be included in differential diagnosis are collagen vascular disease, hyper-eosinophilic syndrome, drug reaction and infection with parasitic organism with definitely no lymphoproliferative disorder and sarcoidosis. As far as IgG4-related lung disease, finding of high IGE, hypocomplementemia, and marked tissue eosinophilia are not specific for IgG4-related diseases and also, tissue shows no features of obliterative phlebitis and storiform fibrosis $[27,28]$ but it can be included in differential diagnosis with high IgG4: IgG ratio $[29,30]$.

\section{Discussion and Conclusion}

Sometimes, we have very interesting and challenging cases where we don't have a definite diagnosis in spite of different possible investigations and collaborating with other health care professionals. We are not sure whether it is appropriate to proceed further with investigating this case or to wait until the patient completes her treatment for the presumptive diagnosis of organizing pneumonia before doing further investigations. It is also important to consider what could be next after lung biopsy with good tissue samples. We currently contemplate if it is better to stop here because doing more and more can cause more harm to a patient than good.

Early diagnosis of IgG4 related diseases is essential as the overall response rate to the first line of treatment with steroids is $93 \%$ and secondly, it prevents complications like fibrosis, periaortitis, and renal failure. Therefore, IgG4 related lung diseases should be considered early in the differential diagnosis of interstitial lung disease associated with autoimmune diseases, bronchiolitis, hyper- eosinophilic syndrome, neoplasms, or sarcoidosis. So, when suspected, as an initial test serum protein electrophoresis and IgG subclass evaluation should be performed as a markedly elevated IgG4>5 g/L (normal level $1.5-5 \mathrm{~g} / \mathrm{L}$ ) is $90 \%$ specific for IgG4 related diseases. For the definite diagnosis of IgG4 related diseases, histopathological findings; a dense lymphoplasmacytic infiltrate, storiform fibrosis, and obliterative phlebitis, are the critical features. The presence of these findings with mild diffuse eosinophilia is strongly suggestive if accompanied by increased numbers of IgG4+ plasma cells in affected tissue to 30-50 lgG4+/ $\mathrm{HPF}$ and IgG4+/IgG+ plasma cell ratio $>40 \%$. 


\section{Conflict of Interest}

Author declare no conflict of interest and consent has been obtained from the patient.

\section{References}

1 Wu W, Bleecker E, Moore W, Busse WW, Castro M, et al. (2014) Unsupervised phenotyping of Severe Asthma Research Program participants using expanded lung data. J Allergy Clin Immunol 133: $1280-1288$.

2 Miranda C, Busacker A, Balzar S, Trudeau J, Wenzel SE (2004) Distinguishing severe asthma phenotypes: role of age at onset and eosinophilic inflammation. J Allergy Clin Immunol 113: 101-108.

3 Haldar P, Pavord ID, Shaw DE, Berry MA, Thomas M, et al. (2008) Cluster analysis and clinical asthma phenotypes. Am J Respir Crit Care Med 178: 218-224.

4 Amelink M, De Groot JC, De Nijs SB, Lutter R, Zwinderman AH, et al. (2013) Severe adult-onset asthma: a distinct phenotype. J Allergy Clin Immunol 132: 336-341.

5 Cordier JF (2006) Cryptogenic organizing pneumonia. Eur Respir J 28 422-446.

6 Epler GR (2001) Bronchiolitis obliterans organizing pneumonia. Arch Intern Med 161: 158.

7 RyhJh, MyersJL, SwensenSJ (2003)Bronchiolardisorders. AmJ Respir Crit Care Med168: 1277.

8 Grinblat J, Mechlis S, Lewitus Z (1981) Organizing pneumonia-like process: An unusual observation in steroid responsive cases with features of chronic interstitial pneumonia. Chest 80: 259-263.

9 Alasaly K, Muller N, Ostrow DN, Champion P, FitzGerald JM (1995) Cryptogenic organizing pneumonia. A report of 25 cases and a review of the literature. Medicine 74: 201-211.

10 Saito Z, Kaneko Y, Hasegawa T, Yoshida M, Odashima K, et al. (2019) Predictive factors for relapse of cryptogenic organizing pneumonia. BMC Pulm Med 19: 10.

11 Onishi Y, Kawamura T, Nakahara Y, Kagami R, Sasaki S, et al. (2017) Factors associated with the relapse of cryptogenic and secondary organizing pneumonia. Respir Investig 55: 10-15.

12 Tomassetti S, Ryu JH, Piciucchi S, Chilosi M, Poletti V (2016) Nonspecific interstitial pneumonia: what is the optimal approach to management?. In Sem Respir Crit Care Med 37: 378-394.

13 Yousem SA. (2017) Cicatricial variant of cryptogenic organizing pneumonia. Hum Pathol 64: 76-82.

14 Umehara $H$, Okazaki K, Masaki $Y$, Kawano $M$, Yamamoto $M$, et al. (2012) Comprehensive diagnostic criteria for IgG4-related disease (IgG4-RD), 2011. Mod Rheumatol 22: 21-30.

15 Mann S, Seidman MA, Barbour SJ, Levin A, Carruthers M, et al. (2016) Recognizing IgG4-related tubulointerstitial nephritis. Can J Kidney Health Dis 3: 34.

16 Pham TH, Damera G, Newbold P, Ranade K (2016) Reductions in eosinophil biomarkers by benralizumab in patients with asthma. Respir Med 111: 21-29.

\section{Acknowledgment}

The author thanks Harjot malhi (Bachelor of Science, Pharmacy $3^{\text {rd }}$ year) for his contribution to manuscript.

17 Bleecker ER, FitzGerald JM, Chanez P, Papi A, Weinstein SF, et al. (2016) Efficacy and safety of benralizumab for patients with severe asthma uncontrolled with high-dosage inhaled corticosteroids and long-acting $\beta 2$-agonists (SIROCCO): A randomised, multicentre, placebo-controlled phase 3 trial. The Lancet 388: 2115-2127.

18 Nair P, Wenzel S, Rabe KF, Bourdin A, Lugogo NL, et al. (2017) Oral glucocorticoid-sparing effect of benralizumab in severe asthma. $\mathrm{N}$ Engl J Med 376: 2448-2458.

19 Zhou Y, Wang L, Huang M, Ding J, Jiang H, et al. (2019) A long-term retrospective study of patients with biopsy-proven cryptogenic organizing pneumonia. Chron Respir Dis 16: 1479973119853829.

20 Shen L, Liu J, Huang L, Zhang Y, Xiao X, et al. (2019) Cryptogenic organizing pneumonia presenting as a solitary mass: Clinical, imaging, and pathologic features. Med Sci Mon Int Med J Exp Clin Res. 25: 466.

21 Hao M, Liu M, Fan G, Yang X, Li J (2016) Diagnostic value of serum IgG4 for IgG4-related disease: A PRISMA-compliant systematic review and meta-analysis. Medicine 2: 95.

22 Zeiger RS, Schatz M, Dalal AA, Chen W, Sadikova E, et al. (2017) Blood eosinophil count and outcomes in severe uncontrolled asthma: a prospective study. J Aller Cl Imm-Pract 5: 144-153.

23 Feinstein MB, DeSouza SA, Moreira AL, Stover DE, Heelan RT, et al. (2015) A comparison of the pathological, clinical and radiographical, features of cryptogenic organizing pneumonia, acute fibrinous and organising pneumonia and granulomatous organizing pneumonia. J Clin Pathol 68: 441-447.

24 Hu X, Yi ES, Ryu JH (2015) Diffuse aspiration bronchiolitis: Analysis of 20 consecutive patients. Jornal Brasileiro de Pneumologia 41: 161166.

25 Carruthers MN, Khosroshahi A, Augustin T, Deshpande V, Stone JH (2015) The diagnostic utility of serum IgG4 concentrations in IgG4related disease. Ann Rheum Dis 74: 14-18.

26 Radzikowska E, Wiatr E, Langfort R, Bestry I, Skoczylas A, et al. (2017) Cryptogenic organizing pneumonia-Results of treatment with clarithromycin versus corticosteroids-An observational study. PloS one 12: e0184739.

27 Corcoran JP, Culver EL, Anstey RM, Talwar A, Manganis CD, et al. (2017) Thoracic involvement in IgG4-related disease in a UK-based patient cohort. Respir Med 132: 117-121.

28 Matsui S, Yamamoto H, Minamoto S, Waseda Y, Mishima M, et al. (2016) Proposed diagnostic criteria for IgG4-related respiratory disease. Respir Investig 54: 130-132.

29 Bledsoe JR, Della-Torre E, Rovati L, Deshpande V (2018) IgG4-related disease: A review of the histopathologic features, differential diagnosis, and therapeutic approach. APMIS 126: 459-476.

30 Masaki Y, Matsui S, Saeki T, Tsuboi H, Hirata S, et al. (2016) A multicenter phase II prospective clinical trial of glucocorticoid for patients with untreated IgG4-related disease. Mod Rheumatol 27: 849-854. 